\title{
Successful therapeutic plasma exchange in cold autoimmune hemolytic anemia in patient with disseminated tuberculosis
}

\author{
N. Belfeki ${ }^{1}$, A. Strazzulla1, P. Isnard ${ }^{2}$, S. Hamrouni', B. Maamar ${ }^{3}$, \\ P. Soulier ${ }^{4}$, M. Monchi ${ }^{4}$, S. Diamantis ${ }^{1}$ \\ ${ }^{1}$ Department of Internal Medicine, Groupe Hospitalier Sud lle de France, Melun, France; \\ 2Department of Pathology, Hôpital Necker des Enfants Malades, Paris, France; \\ ${ }^{3}$ Department of Microbiology, Hôpital Avicenne, Bobigny, France; ${ }^{4}$ Department of Intensive care, \\ Groupe Hospitalier Sud lle de France, Melun, France
}

\section{SUMMARY}

An association of autoimmune hemolytic anemia with disseminated tuberculosis is an exceedingly rare entity. We describe herein a case of cold hemolytic autoimmune anemia associated with miliary tuberculosis resolved with blood transfusions, therapeutic plasma exchange, and antituberculous agents. We discuss the advantages of therapeutic plasma exchange at an early stage in the management of this condition.

Key words: Autoimmune hemolytic anemia, tuberculosis, therapeutic plasma exchange.

\section{INTRODUCTION}

A utoimmune hemolytic anemia (AIHA) has an estimated incidence of 1-3 cases per 100,000 subjects per year. It can occur as an idiopathic disorder or can coexist with another disease, mainly an infectious disease (1). AIHA is one of the rare presentations of tuberculosis (2). Infection caused by Mycobacterium tuberculosis may lead to an immune response that results in the production of $\mathrm{IgG}$, IgM, or both antibodies. This would lead to the mediation of warm, cold, or mixed types of AIHA. The management of this rare hematological complication is a real challenge because of a lack of recommendations: several observations have highlighted the importance of antituberculous agents, blood transfusions, and corticosteroids but the response is not completely satisfactory (3). Therapeutic plasma exchange (TPE) has been used in the treatment of several autoimmune diseases. This therapy would seem logical for cold AIHA, as IgM resides exclusively within the plasma compartment and therefore is expected to be removed efficiently by the exchange process $(4,5)$.
We report herein the case of a 34-year-old female patient presenting with cold AIHA associated with disseminated tuberculosis successfully treated with TPE at the acute phase.

\section{CASE REPORT}

A 34-year-old, pregnant female patient with 32-week amenorrhea and without a significant past medical history was referred to the emergency room for prolonged fever, fatigue, and shortness of breath in the last week. At physical examination, high temperature at $38.5^{\circ} \mathrm{C}$, mucocutaneous pallor, tachycardia with heart beats at 100 per minute, respiratory rate at 20 cycles per minute, and blood pressure at $110 / 70 \mathrm{mmHg}$ were found. Abdominal examination showed spleen enlargement. Obstetrical assessment did not reveal any acute complications related to the fetus or the placenta. Laboratory findings showed a regenerative normocytic normochromic anemia of $6 \mathrm{~g} / \mathrm{dL}$, white cells of 7.0 Giga/L, lymphopenia of $270 / \mathrm{mm}^{3}$, and platelet count of $120 \mathrm{Giga} / \mathrm{L}$. Liver tests showed increased alanine transaminase, $\overline{\text { Corresponding author: }}$ Nabil Belfeki

Department of Internal Medicine Groupe Hospitalier Sud lle de France 270 avenue Marc Jacquet, 77000 Melun, France E-mail: belfeki.nabil@gmail.com 


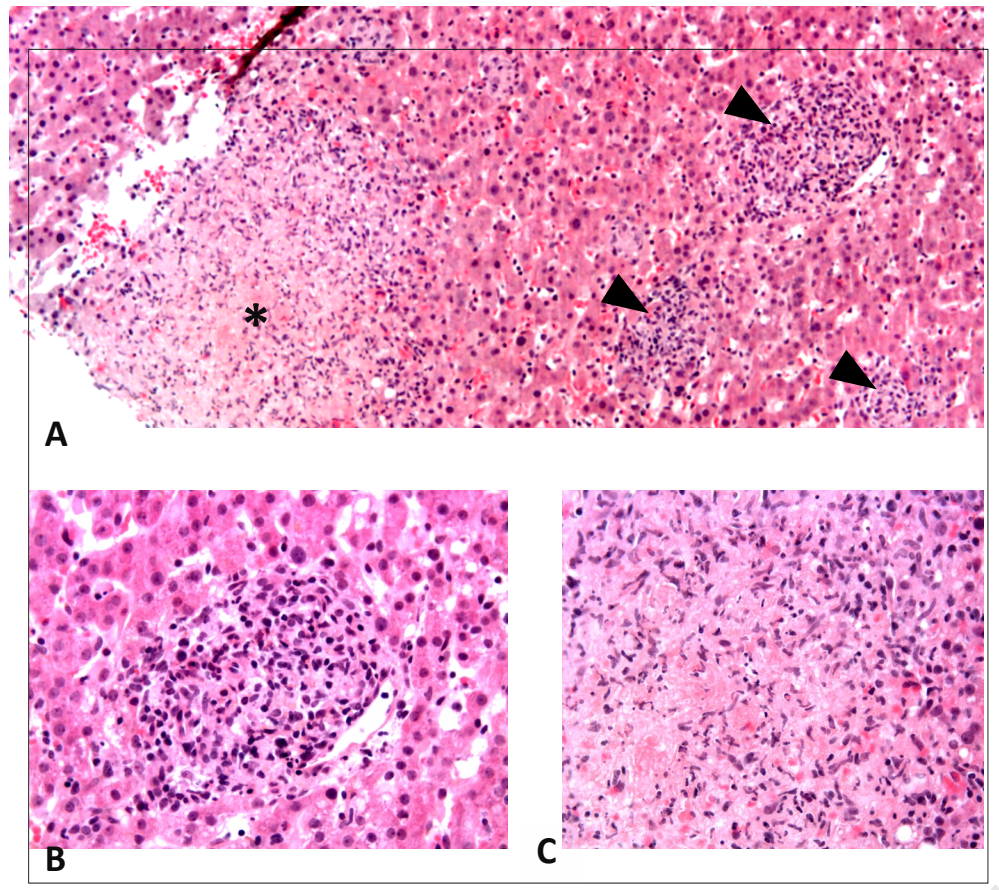

Figure 1 - A) Light microscopy ( $\times 200$ ) using HES staining showed multiple epithelioid granulomatous lesions (black arrow) with focally central necrotic region (asterisk) within the hepatic parenchyma. B) Light microscopy ( $\times 400)$ using HES showing non necrotizing granuloma. C) Light microscopy ( $\times 400)$ using HES showing necrotizing granuloma.

aspartate transaminase, alkaline phosphatase, and gamma glutamyltransferase. Total bilirubin and lactate dehydrogenase were elevated. Haptoglobin was low. Pe-

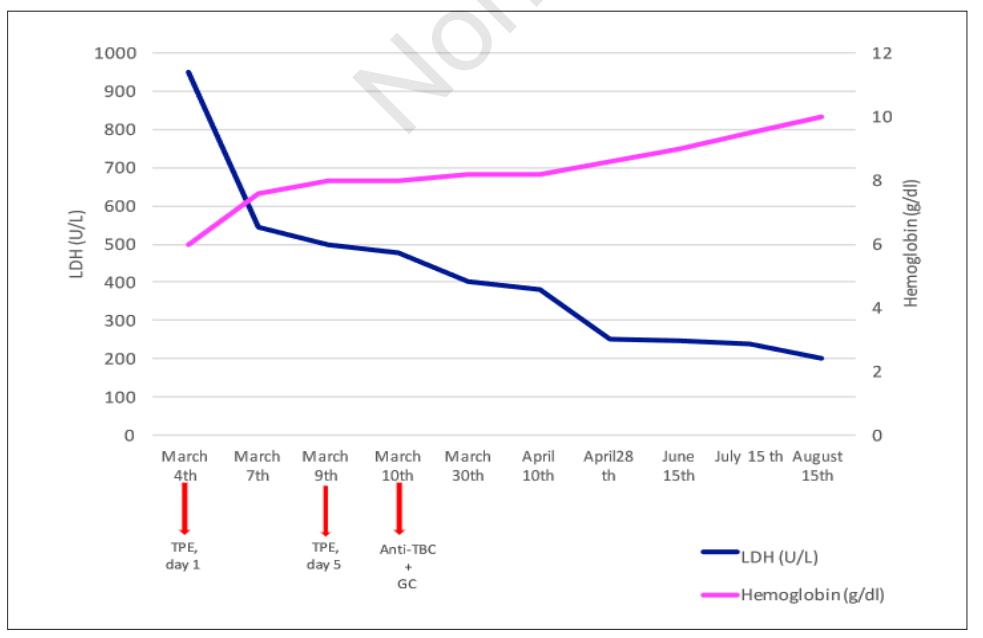

Figure 2 - Graphic representation of patient's lactate dehydrogenase (LDH) and hemoglobin concentrations in relation to the onset and termination of blood transfusions, therapeutic plasma exchange, antituberculous agents and glucocorticoids. ripheral blood smear did not show schizocytes. C-reactive protein was high at 120 $\mathrm{mg} / \mathrm{dL}$ and renal function was normal. Urine and blood bacteriological cultures, as well as vaginal swab, were negative. The indirect antiglobulin test was negative and the direct antiglobulin test was positive with $\mathrm{C} 3 \mathrm{~d}$ class autoantibodies. No alloantibodies were found in the peripheral blood. The patient received blood transfusions with poor results and remained febrile. Fetal extraction was then decided. Afterwards, the patient was referred to the internal medicine department. Further investigations, including antinuclear antibodies, hepatitis B, C and HIV serological tests, were negative. The thoracoabdominal CT-scan showed bilateral interstitial lung disease, thoracic and abdominal lymph nodes enlargement, hepatomegaly, and splenomegaly.

Liver biopsy showed multiple epithelioid granulomatous lesions with focal central necrotic region within the parenchyma (Figure 1). Tissue polymerase chain reaction was positive for Mycobacterium tuberculosis. A diagnosis of autoimmune hemolytic anemia in a patient with miliary tuberculosis was established. TPE with fresh frozen plasma fluid replacement started under intensive care monitoring, five times on five consecutive days. At the end of the $5^{\text {th }}$ day of the TPE session, we observed a steady improvement of hemolytic markers and the patient became persistently independent of blood transfusions for the first time after her admission in the hospital (Figure 2). Anti-tuberculous therapy, consisting of isoniazid 300 $\mathrm{mg} /$ day, rifampicin $600 \mathrm{mg} /$ day, ethambutol $2000 \mathrm{mg} / \mathrm{day}$, and pyrazinamide 2000 $\mathrm{mg} /$ day, was instituted as well as a 4-week course of oral prednisone at the end of the TPE sessions because of persistent hypoxemia due to interstitial lung disease. The patient's general condition improved progressively. The fever discontinued 10 days after antituberculous agents were initiated. Ethambutol and pyrazinamide were stopped after two months and therapy was completed after one year with rifampicin and isoniazid. 


\section{DISCUSSION AND CONCLUSIONS}

Hematological disorders reported in tuberculosis are rare and can affect all lineages. Several mechanisms for the development of anemia in mycobacterial infection include chronic inflammation, iron deficiency, undernutrition, and immune marrow suppression. AIHA is diagnosed on suggestive clinical presentation of hemolysis and laboratory findings compatible with anemia. Direct antiglobulin tests detecting autoantibody and/or complement on the surface of red blood cells confirm the autoimmune character. Agglutination of autoantibodies with an optimum temperature of $37^{\circ} \mathrm{C}$ (DAT IgG type) are called warm autoantibodies AIHA, while agglutination with optimum temperature of 3 to $4^{\circ} \mathrm{C}$ (DAT IgM and/or C3d type) can cause symptoms related to agglutination of red blood cells in cooler parts of the body (6). Besides, cold agglutinin AIHA can cause blood vessel obstruction through the interaction of complement system activation with the coagulation. This could explain placental ischemia and the consequent obstetrical complications (7). We report herein the case of a 34-year- old female patient with no relevant clinical history presenting with impaired general conditions and autoimmune hemolytic anemia developed during the third trimester of pregnancy. Classical complications related to pregnancy such as thrombotic microangiopathy, HELLP (hemolysis, elevated liver enzymes and low platelets count) syndrome, and maternofetal infection were ruled out. The patient remained dependent on blood transfusion and febrile, without an evident etiology. Thus, fetal extraction was decided because of the risk of sudden fetal death (8). Infections account for the major bulk of secondary cold AIHA. Tuberculosis-induced AIHA is a rare entity with only 21 case reports in the published literature (3). The exact etiopathogenesis is not known. However, in experimental animal models, Berkowitz et al. demonstrated that the injection of tuberculous bacilli or their products caused hemolytic anemia, pancytopenia, and myelofibrosis (9). In humans, we suppose that autoimmune cytopenia is related to intrinsic immunological tolerance breakdown due to inflammatory cytokines generated by tuberculous infection. Further studies are needed to elucidate the exact role of Mycobacterium

Table I - Review of the literature with case reports of tuberculosis-associated autoimmune hemolytic anemia.

\begin{tabular}{|l|c|c|c|c|c|c|}
\hline Authors & Year & Country & Age/sex & TB localization & AlHA type & AlHA treatment \\
\hline Siribaddane (10) & 1997 & Sri Lanka & $24 / F$ & Lymph node & Cold agglutinin & ATT \\
\hline Turgut (11) & 2002 & Turkey & $28 / \mathrm{F}$ & Lungs & NS & ATT \\
\hline Kuo (12) & 2001 & Taiwan & $26 / \mathrm{M}$ & Disseminated & ATT \\
\hline Blanche (13) & 2000 & France & $42 / \mathrm{M}$ & Disseminated & Mixed & Steroids, ATT, surgery \\
\hline Abba (14) & 2002 & Saudi Arabia & $21 / \mathrm{F}$ & Gastro intestinal & Mixed & ATT, transfusion \\
\hline Bakshi (15) & 2004 & India & $8 / \mathrm{F}$ & Disseminated & Mixed & Steroids, ATT, transfusion \\
\hline Gupta(16) & 2005 & India & $8 / \mathrm{M}$ & Abdominal & NS & Steroids, ATT \\
\hline Khemiri (17) & 2009 & Tunisia & $11 / \mathrm{F}$ & Lungs & Warm & ATT \\
\hline Nandennavar (18) & 2001 & India & $19 / \mathrm{F}$ & Lymph node & ATT \\
\hline Wu (19) & 2012 & China & $24 / \mathrm{F}$ & Disseminated & Cold & AT, transfusion \\
\hline Safe (20) & 2013 & Brazil & $18 / \mathrm{F}$ & Lungs & Warm & ATT, transfusion \\
\hline Bahbahani (21) & 2014 & Kuwait & $24 / \mathrm{F}$ & Lymph node & Warm & ATT, steroids, Transfusion \\
\hline Anurag (22) & 2015 & India & $25 / F$ & Pulmonary & Cold & ATT, transfusion \\
\hline Shamshad (23) & 2017 & Pakistan & $68 / F$ & Pulmonary & Cold & ATT \\
\hline Our case & 2020 & France & $34 / F$ & Disseminated & Cold & TPE, ATT, steroids, transfusion \\
\hline
\end{tabular}

ATT, anti-tuberculous treatment; NS, non-specified; TPE, therapeutic plasma exchange. 
tuberculosis in AIHA. In a review of the literature of AIHA in patients with tuberculosis, treatment consisted of anti-tuberculosis agents in all cases, blood transfusions in half of them, and glucocorticoids in $38 \%$ of cases mostly in disseminated tuberculosis (3). Table I (10-23) summarizes the published case reports of tuberculosisassociated autoimmune hemolytic anemia. For infection-associated hemolytic anemia, immediate initiation of an appropriate antimicrobial therapy is the cornerstone of the treatment. In the literature, glucocorticoids were used in case of warm and/or mixed AIHA in association with anti-tuberculosis agents and mainly in cases of disseminated tuberculosis $(13,15,21)$.

However, glucocorticoids and splenectomy are not effective in the majority of patients with cold AIHA, in contrast to warm AIHA (24). The reported effectiveness of steroids or splenectomy in cold AIHA related to tuberculosis is probably due to the fact that patients with IgG cold reacting antibodies, antibodies with a higher thermal amplitude that cause some warm hemolysis, or mixed warm and cold AIHA were included. In fact, glucocorticoids will downregulate phagocytosis, but will not discontinue antibody production. Thus, reported satisfactory response is around 10 to $15 \%(25,26)$. Besides, splenectomy is not recommended because the intratissular hemolysis site in cold agglutinin AIHA is the liver. No targeted rituximab therapy was reasonable at this early stage with high suspicions of an underlying infectious process. For critical hemolysis, when it is unacceptable to wait several days before antituberculous agents take effect, therapeutic plasma exchange is thought to be highly effective in removing cold agglutinins because these are always IgM antibodies and occupy the intravascular sector $(27,28)$. Moreover, according to the American Society for Apheresis, therapeutic apheresis for severe cold agglutinin disease is considered a second-line therapy either as stand-alone treatment or in conjunction with other treatments (29). This procedure does not address the source of antibody production and thus must be considered as a temporizing therapeutic alter- native until the underlying cause is under control. To the best of our knowledge, this is the first case of cold AIHA in a patient with disseminated tuberculosis treated with therapeutic plasma exchange with a favorable outcome. Larger studies are mandatory to confirm the indication of TPE in cold AIHA.

\section{Conflict of interests}

The authors declare no potential conflict of interests.

\section{REFERENCES}

1. Nandennavar M, Cyriac S, Krishnakumar TG. Immune haemolytic anaemia in a patient with tuberculous lymphadenitis. J Glob Infect Dis. 2011; 3: 89.

2. Lucchini G, Masera N, Foti G, et al. A lifethreatening paediaric case of acute autoinmune haemolytic anaemia (AIHA) successfully cured by plasma-exchange and combined immunosuppressive treatment. Transfus Apheresis Sci. 2009; 40: 115.

3. Rathish D, Siribaddana S. Tuberculosis induced autoimmune haemolytic anaemia: a systematic review to find out common clinical presentations, investigation findings and the treatment options. Allergy Asthma Clin Immunol. 2018; 26: 11.

4. Cerdas-Quesada C. A life-threatening case of autoimmune hemolytic anemia successfully treated by plasma-exchange. Transfus Apher Sci. 2010; 42: 235-7.

5. Gil-Santana L, Cruz Lab, Arriaga MB, et al. Tuberculosis-associated anemia is linked to a distinct inflammatory profile that persists after initiation of antitubercular therapy. Sci Rep. 2019; 4: 1381.

6. Theodosia A. Warm antibody autoimmune hemolytic anemia. Hematology Am Soc Hematol Educ Program. 2016; 2: 690-7.

7. Maroto A, Martinez-Diago C, Tio G, et al. Autoimmune hemolytic anemia in pregnancy: a challenge for maternal and fetal follow-up. J Matern Fetal Neonatal Med. 2020; 25: 1-3.

8. Koike Y, Akiyama Y, Utani A. Cold agglutinin disease associated digital gangrene treated with plasmapheresis. Indian J Dermatol Venereol Leprol. 2014; 80: 575-6.

9. Berkowitz FE. Hemolysis and infection: categories and mechanisms of their interrelationship. Rev Infect Dis. 1991; 13: 1151-62.

10. Siribaddana SH, Wijesundera A. Autoimmune hemolytic anemia responding to antituberculous treatment. Trop Doct. 1997; 23: 243-4.

11. Turgut M, Usun O, Kelkytly E, Ozer O. Pulmonary tuberculosis associated with autoim- 
mune hemolytic anemia: An unusual presentation. Turk J Haematol. 2002; 19: 477-80.

12. Kuo PH, Yang PC, Kuo SS, Luh KT. Severe immune hemolytic anemia in disseminated tuberculosis with response to antituberculosis therapy. Chest. 2001; 119: 1961-3.

13. Blanche P, Rigolet A, Massault PP, et al. Autoimmune haemolytic anaemia revealing military tuberculosis. J Infect. 2000; 40: 292.

14. Abba AA, Laajam MA, Al Majid FM. Autoimmune hemolytic anemia associated with intestinal tuberculosis. Ann Saudi Med. 2002; 22: 68-9.

15. Bakhshi S, Rao IS, Jain V, Arya LS. Autoimmune hemolytic anemia complicating disseminated childhood tuberculosis. Indian J Pediatr. 2004; 71: 549-51.

16. Gupta V, Bhatia BD. Abdominal tuberculosis with autoimmune hemolytic anemia. Indian $\mathrm{J}$ Pediatr. 2005; 72: 175-8.

17. Khemiri M, Zouari S, Barsaoui S. Autoimmune bicytopenia in pulmonary tuberculosis: Report of a pediatric case. Respir Med CME. 2008; 1: 281-3.

18. Nandennavar M, Cyriac S, Krishnakumar, Sagar T. Immune hemolytic anemia in a patient with tuberculous lymphadenitis. J Glob Infect Dis. 2001; 3: 89-91.

19. Wu B, Rong R. Cold agglutinin syndrome with severe haemolytic anaemia in a patient diagnosed of disseminated tuberculosis and concomitant Mycoplasma pneumoniae infection. Transfus Med. 2012; 22: 151-2.

20. Safe IP, O'Brien C, Ferreira FR, et al. Tuberculosis associated with transient hemolytic anemia responsive to tuberculosis chemotherapy: A case report. Braz J Infect Dis. 2013; 17: 110-11.
21. Bahbahani H, Al-Rashed M, Almahmeed M. Tuberculosis and autoimmune hemolytic anemia: Case report and literature review. J Appl Hematol. 2014; 5: 164-7.

22. Anurag L, Choudhary R. Cold agglutinin induced hemolytic anemia in a patient with pulmonary tuberculosis. Int J Med Res Health Sci. 2015; 4: 911-2.

23. Shamshad GU, Salamat N, Umair M. Cold agglutinin disease in a patient of pulmonary tuberculosis. J Coll Physicians Surg Pak. 2017; 27: 92-4.

24. Berentsen S. How I manage patients with cold agglutinin disease In case of AIHA. Br J Haematol. 2018; 181: 320.

25. Berentsen S, Ulvestad E, Langholm R. Primary chronic cold agglutinin disease: a population based clinical study of 86 patients. Haematologica. 2006; 91: 460.

26. Berentsen S. Cold agglutinin disease. Hematology Am Soc Hematol Educ Program 2016; 2: 226-31.

27. Zoppi M, Oppliger R, Althaus U, Nydegger U. Reduction of plasma cold agglutinin titers by means of plasmapheresis to prepare a patient for coronary bypass surgery. Infusions Ther Transf Med. 1993; 20: 19.

28. Pereira A, Mazzara R, Escoda L. Anti-Sa cold agglutinin of IgA class requiring plasma-exchange therapy as early manifestation of multiple myeloma. Ann Hematol. 1993; 66: 315.

29. Padmanabhan A, Connelly-Smith L, Aqui N. Guidelines on the use of therapeutic apheresis in clinical practice - evidence-based approach from the Writing Committee of the American Society for Apheresis: The Eighth Special Issue. J Clin Apher. 2019; 34: 171-354. 